\title{
Trazabilidad de los registros de mamíferos en el marco del otorgamiento de licencias ambientales para proyectos de hidrocarburos en Colombia
}

\author{
Paul Andrés Gómez-Sandoval ${ }^{1, *}$, José Iván Mojica-Corzo² ${ }^{2}$ Ofelia Mejía-Egas ${ }^{3}$ \\ ${ }^{1}$ Departamento de Biología, Facultad de Ciencias, Universidad Nacional de Colombia, Bogotá, Colombia \\ ${ }^{2}$ Instituto de Ciencias Naturales, Universidad Nacional de Colombia, Bogotá, Colombia \\ ${ }^{3}$ Departamento de Biología, Facultad de Ciencias, Universidad del Cauca, Popayán, Colombia
}

\begin{abstract}
Resumen
Se evaluó la trazabilidad de los registros de mamíferos informados en el marco del otorgamiento de licencias ambientales en el sector de hidrocarburos en Colombia, en una muestra de 43 proyectos con licencias otorgadas en el 2014. El análisis se hizo mediante un método propuesto por los autores, el cual se fundamenta en el concepto de voucher biológico. Los resultados demostraron la falta de evidencia biológica verificable, que permita poner a prueba o revisar la identidad y características de la fauna de mamíferos reportada. Bajo estas circunstancias, la información pierde confiabilidad, lo cual limita la posibilidad de usarla, revisarla o reinterpretarla en investigaciones futuras. Si esta condición resulta ser un rasgo común en las caracterizaciones de la fauna que se presentan para el otorgamiento de licencias a los proyectos, ello implicaría que se está desperdiciando una oportunidad única para el conocimiento de la diversidad biológica del país, y que las decisiones sobre la viabilidad ambiental de los proyectos de hidrocarburos estarían respaldadas por estudios de mamíferos con un grado significativo de incertidumbre, en cuanto a la identidad y las características de los taxones registrados. (C) 2017. Acad. Colomb. Cienc. Ex. Fis. Nat.
\end{abstract}

Palabras clave: Fauna de mamíferos; Estudios de impacto ambiental; Caracterización; Inventario; Evaluación del impacto ambiental; Industria del petróleo; Voucher.

\section{Traceability of mammal records in the framework of environmental licensing of oil projects in Colombia}

\begin{abstract}
We evaluated the traceability of mammalian records reported in the framework of environmental licensing in the oil sector in Colombia based on a sample of 43 projects licensed during 2014. Our analysis was done using the method we have proposed based on the concept of biological voucher. Our results showed a lack of the verifiable biological evidence that allows to test or review the identity and features of reported mammals. Under these circumstances, the information loses reliability and its eventual use, review or reinterpretation in future research work is restricted. If this condition proves to be a common feature in the studies of fauna presented for projects licensing, it would imply that a unique opportunity to increase the knowledge on the country's biological diversity is being wasted, and that decision-making on environmental viability of oil projects is supported on mammalian studies with a significant degree of uncertainty in terms of the identity and characteristics of the recorded taxa. (C) 2017. Acad. Colomb. Cienc. Ex. Fis. Nat.
\end{abstract}

Key words: Mammals; Environmental impact statement; Inventory; Environmental impact assessment; Oil industry; Voucher.

\section{Introducción}

En los últimos años en Colombia, la Autoridad Nacional de Licencias Ambientales (ANLA) ha otorgado anualmente cerca de un centenar de nuevas licencias a proyectos de hidrocarburos (ANLA, 2012, 2013, 2014, 2015), lo que lo convierte en uno de los sectores con mayor representación en términos de trámites ante esta entidad.

Como parte de los Estudios de Impacto Ambiental (EIAs) requeridos para el otorgamiento de licencias de estos proyectos (Decreto 1076 de 2015), se exigen caracterizaciones o inventarios biológicos, con los que en términos generales se busca estimar el estado de algunos atributos de la biota (e.g. riqueza, composición, amenazas) que podría verse afectada por la ejecución de estas actividades.

\footnotetext{
*Correspondencia:

Paul Andrés Gómez-Sandoval, pagomezs@unal.edu.co

Recibido: 25 de noviembre de 2016

Aceptado: 25 de febrero de 2017
} 
La información biótica incluida en los EIAs presentados para elotorgamiento de licencias ambientales de proyectos en el mundo, viene siendo sometida a exámenes críticos (e.g. Lee \& Colley, 1990, Atkinson, et al., 2000, Mandelik, et al., 2005, Bataineh, 2007, Khera \& Kumar, 2010, Hallat, et al., 2015), que han revelado debilidades latentes en cuanto a la calidad y suficiencia de la información. Esto puede incidir de manera negativa en la toma de decisiones y el propósito de la conservación efectiva de la diversidad biológica, en las áreas de acción de los proyectos. Sin embargo, a escala nacional, no se dispone de publicaciones científicas orientadas a la evaluación de la información biótica que es presentada para el otorgamiento de licencias ambientales de proyectos.

En el marco de las caracterizaciones o inventarios de la biodiversidad, contar con Vouchers o evidencia verificable de los muestreos, resulta crucial porque constituyen la base tangible o intangible para la comprobación de los registros biológicos reportados (i.e. trazabilidad de la información) y garantizan el uso o análisis futuro de la información recolectada (i.e. revisión, reevaluación o reinterpretación; Martin, 1990, Wheeler, 2003, Funk, et al., 2005, Turney, et al., 2015, Schilthuizen, et al., 2015). El voucher biológico es definido como: un espécimen, una muestra, o producto del mismo, y sus datos asociados, que documenta la existencia de un organismo en un lugar y tiempo determinado, de una manera consistente con estandares disciplinarios, para asegurar la repetibilidad de la investigación, que de otra forma no podría ser adecuamente revisada o reevaluada" (Kageyama, et al., 2006: 262).

Bajo este concepto, se puede deducir que la evidencia de un muestreo biótico debe cumplir con tres criterios primarios, para ser catalogada voucher biológico: a) Existencia y repetibilidad: corresponder a un espécimen, muestra o producto del mismo, susceptible de verificación, revisión o evaluación posterior; b) Espacial: tener información asociada del lugar dónde se registró el espécimen, muestra o producto del mismo; c) Temporal: tener información asociada del momento en el que se registró el espécimen, muestra o producto del mismo.

En este contexto, esta investigación tuvo como objetivo el análisis de la trazabilidad de los registros de especies de la mastofauna, que se presentan en el marco del otorgamiento de licencias ambientales de proyectos de hidrocarburos en Colombia. Los mamíferos fueron elegidos como grupo de estudio, debido a la importancia que tienen estos organismos por su uso comercial, recreativo, valor simbólico (e.g. especies bandera o carismáticas; Isasi-Catalá, 2011) y por ser proveedores de servicios y reguladores de dinámicas ecosistémicas (Ceballos \& Erhlich, 2009, Fleming, et al., 2009, Boyles, et al., 2011, Kunz, et al., 2011, MedranoNajera, et al., 2014, Regan, et al., 2015).

\section{Materiales y métodos}

Muestra de la investigación. Se identificaron los proyectos de hidrocarburos que fueron licenciados durante el año
2014 en Colombia, a través de la consulta de los boletines de la Gaceta Ambiental (enero a diciembre), donde la Autoridad Nacional de Licencia Ambientales (ANLA) publica resoluciones, autos, certificaciones y permisos. Se hallaron 86 resoluciones relativas al otorgamiento de licencias ambientales del sector de hidrocarburos, de las cuales 49 correspondieron a nuevas licencias y 37 a modificaciones a las ya existentes.

La muestra de esta investigación fue determinada por aquellos EIAs de las nuevas licencias del sector de hidrocarburos, a los que fue posible acceder a toda su información en formato digital, bajo las directrices establecidas en la Resolución de la ANLA N 0173 del 17 de febrero de 2015, "Por la cual se regula el cobro de copias expedidas por la entidad”. Bajo este criterio, la muestra consolidada se obtuvo de 43 EIAs (i.e. 87.8\% del universo 2014), a partir de los cuáles se efectuó el escrutinio de las caracterizaciones de mamíferos, que fueron el grupo biológico modelo de esta investigación (Información suplementaria, anexo 1, http:// www.raccefyn.co/index.php/raccefyn/article/download SuppFile/439/1809).

La localización geográfica de los proyectos analizados en este estudio, se presenta en la figura 1.

Análisis de datos. Los autores de esta investigación, diseñaron una metodología para analizar la trazabilidad de los registros de mamíferos en el marco del otorgamiento de licencias ambientales (Figura 2), que está fundamentada en el concepto de Voucher biológico (sensu Kageyama, et al., 2006). El objetivo de esta propuesta operativa, fue determinar cuál información registrada en las caracterizaciones, es susceptible de ser catalogada como Voucher biológico de los reportes de la mastofauna y por ende tiene el potencial de ser revisada, reevaluada, refutada, reinterpretada o usada como evidencia científica de investigaciones futuras.

Bajo esta metodología (Figura 2), para cada uno de los 43 estudios de mamíferos que constituyeron la muestra, en primera instancia se identificaron los tipos de registro biológico con los que se obtuvo el reporte de las especies de mamíferos. Posteriormente, se determinó la clase de evidencia que fue proveída para cada tipo de registro. Finalmente, cada clase de evidencia fue sometida a la verificación del cumplimiento de los tres criterios asociados al concepto de Voucher biológico (sensu Kageyama, et al., 2006): a) Criterio de Existencia y repetibilidad: corresponder a un espécimen, muestra o producto del mismo, susceptible de verificación, revisión o evaluación posterior; b) Criterio espacial (i.e. ¿dónde?): tener información asociada del lugar dónde se registró el espécimen, muestra o producto del mismo; c) Temporal (i.e. ¿cuándo?): tener información asociada del momento en el que se registró el espécimen, muestra o producto del mismo.

De esta manera, se discriminó la evidencia que garantiza de manera objetiva y verificable, la trazabilidad de los registros de la mastofauna presentados en el marco del otorgamiento de licencias ambientales de proyectos. 


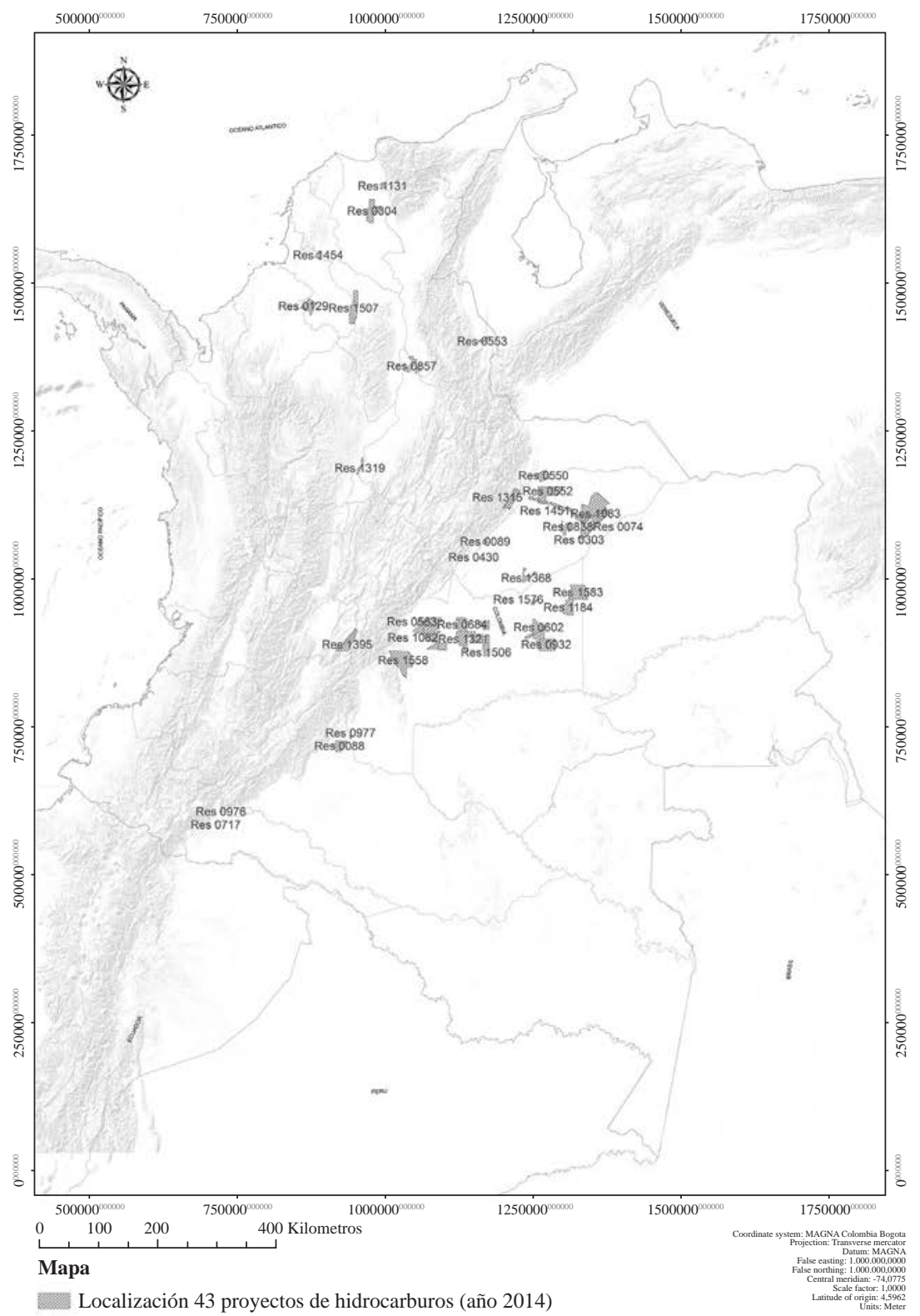

Figura 1. Localización de 43 proyectos de hidrocarburos licenciados en Colombia durante el año 2014, de los que se obtuvo la muestra. Fuente: Elaborado a partir de base cartográfica de los 43 EIAs obtenidos en la ANLA.

\section{Resultados y discusión}

Los 43 proyectos de hidrocarburos que constituyeron la muestra de este trabajo, abarcan zonas de 18 departamentos y 61 municipios de Colombia; concentrándose principalmente en la región Caribe, los Andes centrales y la Orinoquia (Figura 1). Las caracterizaciones de mamíferos desarrolladas para el otorgamiento de licencias ambientales de estos emprendimientos, reportaron en conjunto 231 taxones identificados hasta el nivel de especie (Información suplementaria, anexo 2, http://www.raccefyn.co/index.php/raccefyn/article/ downloadSuppFile/439/1810). La riqueza observada estuvo en un rango de 14 a 70 especies por proyecto, con un promedio de 38,7 y desviación estándar de 14,7.
Entre estos registros, se presentan algunas nominaciones desactualizadas o equívocas (sensu Solari, et al., 2013, Ramírez-Chaves \& Suárez-Castro, 2014, Ramírez-Chaves, et al., 2016; Información suplementaria, anexo 2, http://www. raccefyn.co/index.php/raccefyn/article/downloadSuppFile/ 439/1810): dieciocho (18) taxones reportados (Bassaricyon gabbii, Cebus/Sapajus macrocephalus, Coendou bicolor, Didelphis albiventris, Eptesicus diminutus, Eumops bonariensis, Odocoileus virginianus, Mazama americana, Mazama nemorivaga, Molossus cf. currentium, Neonycteris pusilla, Oryzomys albigularis, Oligoryzomys fulvescens, Proechimys trinitatus, Rhogeessa tumida, Saimiri sciureus, Sigmodon hispidus, Vampyressa pusilla) están excluidos de las listas más recientes de mamíferos del país, debido a que 


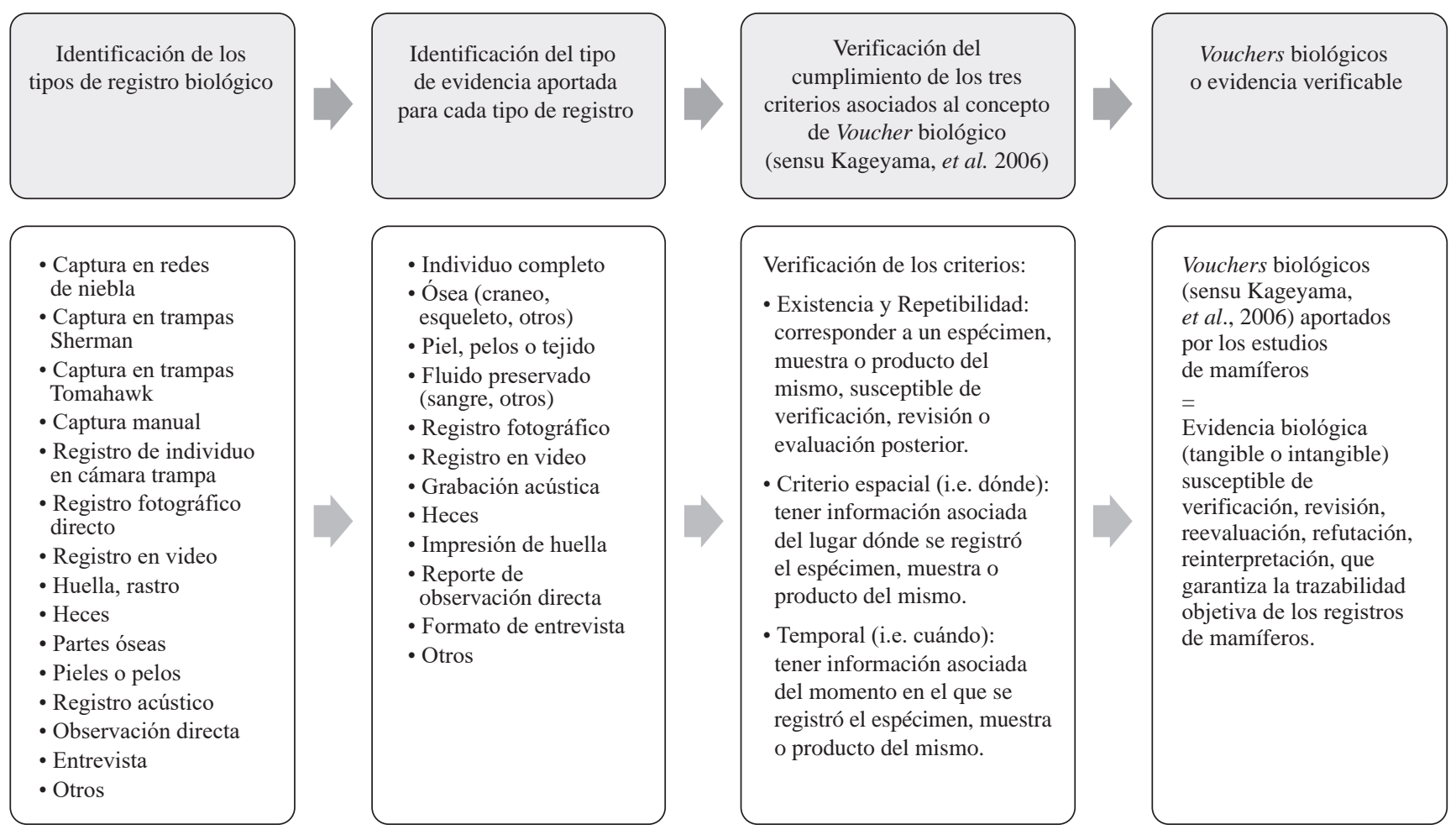

Figura 2. Propuesta metodológica para identificar Vouchers biológicos aportados en los estudios de mamíferos.

Fuente: Propuesta operativa de los autores de esta investigación, a partir del desarrollo teórico de Kageyama, et al. 2006.

la evidencia actual no soporta su presencia en Colombia; los roedores Mus musculus, Rattus norvegicus y Rattus rattus, desde la publicación de Solari, et al., (2013), también se omiten de la mastofauna nativa por corresponder a especies introducidas (a pesar de encontrarse asilvestradas en algunas regiones; Ramírez-Chaves, et al., 2011); veintitrés (23) taxones (Agouti paca, Artibeus anderseni, Artibeus glaucus, Artibeus cf. gnoma, Artibeus intermedius, Artibeus phaeotis, Callicebus ornatus, Callicebus torquatus, Coendou sanctaemartae, Lagothrix lugens, Lasiurus ega, Microsciurus pulcheranii, Mimon crenulatum, Oryzomys bicolor, Oryzomys talamancae, Proechimys urichi, Saguinus fuscicollis, Sciurus granatensis, Sciurus igniventris, Sciurus spadiceus, Tayassu tajacu, Tonatia silvicola, Vampyressa bidens) corresponden a sinónimos de especies válidas para el paísAsí, estos proyectos licenciados en el año 2014, aglutinan registros cercanos al 38\% (i.e. 200 spp válidas) de las especies nativas de mamíferos conocidas para Colombia (sensu RamírezChaves, et al., 2016).

Estas cifras demuestran el potencial que tiene la información proveniente de los Estudios de Impacto Ambiental, en la contribución al conocimiento de los mamíferos y la biodiversidad del territorio colombiano (King, et al., 2012). Estos datos adquieren mayor relevancia, si se considera que algunos de los reportes biológicos están asociados a localidades que no han sido previamente muestreadas en el marco de investigaciones científicas.
La importancia de los registros bióticos obtenidos en el contexto del otorgamiento de licencias ambientales y los proyectos de hidrocarburos en Colombia, se ejemplifica con los trabajos de Suárez-Castro, et al., (2012) y MoralesMartínez \& Suárez-Castro (2014), quiénes a partir de la revisión de ejemplares depositados en colecciones biológicas de referencia científica, que provenían de inventarios de mamíferos realizados para proyectos petroleros, publicaron la ampliación de la distribución conocida para Colombia de los quirópteros Glyphonycteris silvestris y Peropteryx leucoptera, y los primeros registros para el país de Glyphonycteris daviesi y Peropteryx pallidoptera. Estos hallazgos fueron posibles gracias a que se contaba con Vouchers biológicos del muestreo realizado (sensu Kageyama, et al., 2006). La utilidad y confiabilidad de la información obtenida en los estudios bióticos desarrollados para el otorgamiento de licencias ambientales de proyectos en Colombia, está condicionada por su trazabilidad, por la posibilidad de revisar, confirmar, reevaluar o reinterpretar los registros biológicos y su datos asociados (Wheeler, 2003, Funk, et al., 2005, Schilthuizen, et al., 2015)

Tipos de registro biológico y la riqueza observada de mamíferos. La figura 3 presenta el número total de especies nativas de mamíferos, por tipo de registro biológico (sensu Solari, et al., 2013, Ramírez-Chaves \& SuárezCastro, 2014, Ramírez-Chaves, et al., 2016; Información suplementaria, anexo 2, http://www.raccefyn.co/index.php/ 
Rev. Acad. Colomb. Cienc. Ex. Fis. Nat. 41(158):51-58, enero-marzo de 2017 doi: http://dx.doi.org/10.18257/raccefyn.439

raccefyn/article/downloadSuppFile/439/1810). Las entrevistas (110 spp), la captura en redes de niebla (90 spp), las observaciones directas (81 spp) y las huellas y rastros (40 spp), fueron las clases que aportaron más información a la riqueza total observada. En este contexto, es importante destacar que para 37 especies, algunos EIAs no presentaban información acerca del tipo de registro (Figura 3, Información suplementaria, anexo 2, http://www. raccefyn.co/index.php/raccefyn/article/downloadSuppFile/ 439/1810).

Algunos taxones reportados en los estudios de la mastofauna analizados en esta investigación, motivan dudas razonables sobre la veracidad de su registro (Información suplementaria, anexo 2, http:/www.raccefyn.co/index.php/ raccefyn/article/downloadSuppFile/439/1810). Es el caso del murciélago Diaemus youngii, y los roedores Oecomys bicolor y Oecomys concolor, que fueron reportadas únicamente a través de entrevistas. El registro de estas especies exclusivamente por este método genera alta incertidumbre, debido a sus características morfológicas, etología, sitios de refugio y alimentación, que hacen poco probable su conocimiento específico u observación frecuente por parte de los habitantes del área de influencia de estos proyectos.

En este sentido, también resultan inciertos los reportes de tres especies de murciélagos registradas exclusivamente a través de observaciones directas: Choeroniscus minor, Glossophaga commissarisi, Molossus bondae (Información suplementaria, anexo 2, http://www.raccefyn.co/index.php/ raccefyn/article/downloadSuppFile/439/1810).
Aunque las entrevistas constituyen una fuente común de información en las investigaciones de mamíferos (Proulx \& Drescher, 1993, Voss \& Emmons, 1996, PolancoOchoa, et al., 2000, Voss, et al., 2001, Vriesendorp, et al., 2007, Mejía-Correa \& Díaz-Martínez, 2009, Castaño y Corrales, 2010, Pitman, et al., 2014, Liévano-Latorre \& López-Arevalo, 2014), debido a que permiten abordar el conocimiento local, los usos y valores dados a los mamíferos por los habitantes de un área dada (LiévanoLatorre \& López-Arevalo, 2014), este método por si solo no garantiza trazabilidad en los reportes taxonómicos, y por ello, su precisión y confiabilidad suele ser cuestionada (Patton, et al., 1982, Sánchez, et al., 2004, Zapata-Ríos, et al., 2006, MINAM, 2015). Así mismo, bajo el concepto de Voucher biológico (Kageyama, et al., 2006), este método no cumple con el criterio de Existencia y Repetibilidad, porque no hay forma de verificar, revisar o reevaluar, la identidad y características del organismo registrado por estos medios. Estas debilidades son compartidas por los registros obtenidos únicamente a través de observaciones visuales, donde la confiabilidad de la información recae en la experticia del observador y la precisión del reporte o su información asociada, es aceptada a priori y no puede ser puesta prueba.

Tipo de evidencia y trazabilidad de la información de los muestreos de mamíferos. En los 43 estudios examinados, las únicas clases de evidencia ofrecida fueron los registros fotográficos incluidos en los EIAs y los formatos de entrevistas presentados como anexos. Una proporción sustancial de los taxones reportados, carecían de evidencia susceptible de

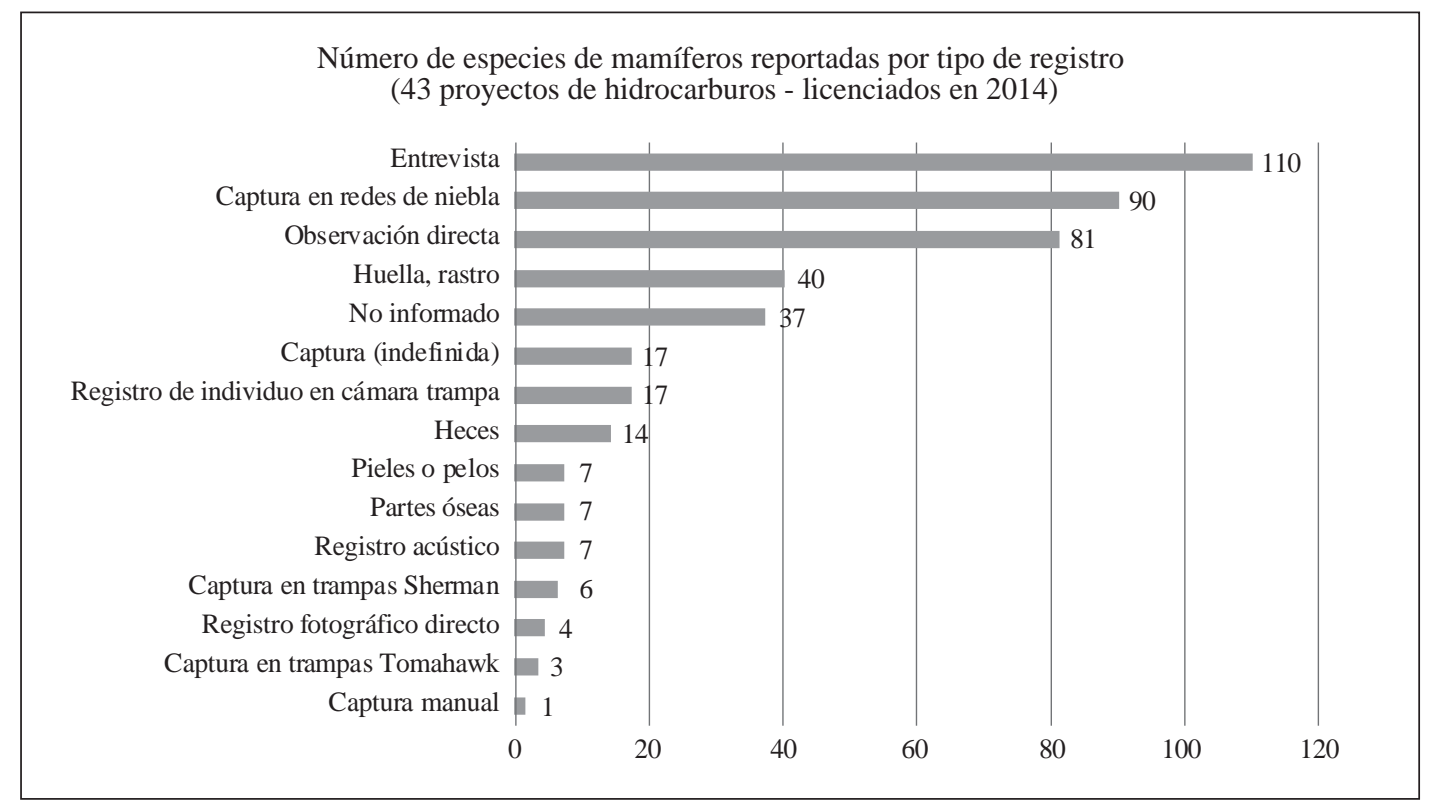

Figura 3. Tipos de registro biológico y su aporte a la riqueza observada, en 43 estudios de mamíferos desarrollados para el licenciamiento de proyectos de hidrocarburos en Colombia. Aclaración: "captura (indefinida)" corresponde a registros en los que no se especifica el tipo de captura realizada (e.g. en trampa Sherman, Tomahawk, manual).

Fuente: Elaboración propia, a partir de la información obtenida de 43 EIAs de proyectos de hidrocarburos licenciados en Colombia, en el año 2014 (detalles en Información suplementaria, anexo 2, http:/www.raccefyn.co/index.php/raccefyn/article/ downloadSuppFile/439/1810). 
verificación (aún la fotográfica). En ninguno de los estudios, se informó sobre la ejecución de recolecta científica o el depósito de algún tipo de Voucher (sensu Kageyama, et al., 2006) en colecciones biológicas de referencia (e.g. individuos completos; partes óseas; pieles, pelos o tejidos; impresión de huellas; heces; fluidos preservados). Así mismo, se encontró que algunos EIAs de la muestra que fueron realizados por la misma empresa consultora, repetían fotografías en sus informes, como sustento del registro de las especies.

Si se tiene en cuenta que las entrevistas y las observaciones directas, están entre los tipos de registro que aportaron más información a la riqueza de especies (Figura 3), y que estas clases de soporte no cumplen con el criterio de Existencia y Repetibilidad para ser considerados Vouchers biológicos (Figura 2; Kageyama, et al., 2006); es posible inferir que un procentaje mayoritario de los reportes de mamíferos contenidos en estos estudios, no están sustentados en evidencia biológica verificable o refutable.

Esta investigación no pretende sugerir que la recolecta científica es la mejor o única alternativa para garantizar trazabilidad, confiabilidad y el uso futuro de los registros obtenidos en las caracterizaciones bióticas. Existen otros tipos de evidencia biológica (e.g. piel, pelos o tejido; fluido preservado: sangre, otros; registro fotográfico; registro en video; grabación acústica; heces; impresiones de huellas), que pueden cumplir a cabalidad como Vouchers biológicos de los muestreos (sensu Kageyama, et al., 2006), sin implicar el sacrificio de los organismos. El aspecto crucial en todos los casos, es aportar información espacial y temporal asociada al registro biótico y la mayor cantidad de evidencia susceptible de verificación, que pueda reunirse, preservarse y consultarse. En este sentido, todo tipo de Voucher biológico obtenido en este tipo de estudios, debería ser depositado en colecciones biológicas de referencia científica, para garantizar su acceso y uso futuro.

\section{Conclusiones}

La mayoría de los estudios de la mastofauna presentados para el otorgamiento de licencia ambiental a los 43 proyectos de hidrocarburos analizados en esta investigación, no brindan evidencia biológica susceptible de verificación (i.e. Vouchers sensu Kageyama, et al., 2006), que permita poner a prueba su veracidad o revisar la identidad y características de los organismos registrados. Bajo estas circunstancias, la información entregada en estos inventarios rápidos pierde confiabilidad y se ve limitada la posibilidad de uso, revisión o reinterpretación en investigaciones futuras (e.g. patrones de distribución y actualización de listas de especies).

Si esta condición resulta ser un rasgo común en las caracterizaciones de la fauna que se presentan para el otorgamiento de licencias ambientales a proyectos en Colombia, se está derrochando una oportunidad única para el conocimiento de la diversidad biológica del país. La información biótica incluida en los EIAs tiene un potencial enorme, por su cobertura espacial en el territorio colombiano, el número de proyectos que se licencian anualmente (e.g. 180 en 2014, según ANLA, 2015) y la singularidad de los sitios dónde se desarrollan los muestreos de la biota.

Los hallazgos de este trabajo sugieren que la toma de decisiones acerca de la viabilidad ambiental de los proyectos de hidrocarburos en Colombia, se hace sobre la base de caracterizaciones de mamíferos que guardan un grado importante de incertidumbre, en términos de la identidad y características de los taxones registrados.

En este sentido, las autoridades ambientales y la comunidad académica, deben dirigir esfuerzos hacia la reglamentación y educación, sobre la necesidad de aportar evidencia biológica verificable en las caracterizaciones bióticas, de manera que se garantice la trazabilidad de la información y su disponibilidad a futuro para la comunidad científica y el público general (Schilthuizen, et al., 2015, Turney, et al., 2015).

Son apremiantes más trabajos de investigación dirigidos a evaluar de manera continua, la calidad de los estudios y la información presentada en el marco del otorgamiento de licencias ambientales (e.g. Lee \& Colley, 1990, Warken \& Buckley, 1998, Gray \& Jones, 1999, Atkinson, et al., 2000, Byron, et al., 2000, Mandelik, et al., 2005, Bataineh, 2007, Khera \& Kumar, 2010, Gómez-Sandoval, 2015; Hallat, et al., 2015). Estos esfuerzos pueden contribuir en los procesos de mejora continua del sistema de otorgamiento de licencias ambientales colombiano y ayudar a detectar puntos débiles en los instrumentos reglamentarios, los procesos de evaluación, la preparación de los EIAs y los métodos comúnmente empleados para el desarrollo de los estudios ambientales.

\section{Información suplementaria}

Anexo 1. Información de 43 proyectos de hidrocarburos licenciados en colombia durante el año 2014. Vea el anexo 1 en: http://www. raccefyn.co/index.php/raccefyn/article/downloadSuppFile/439/1809

Anexo 2. Taxones por tipo de registro biológico, reportados en 43 estudios de mamíferos desarrollados para el licenciamiento ambiental de proyectos de hidrocarburos en Colombia. Vea el anexo 2 en: http://www.raccefyn.co/index.php/raccefyn/article/download SuppFile/439/1810

\section{Conflicto de intereses}

Los autores declaran que no tienen conflicto de intereses.

\section{Agradecimientos}

Expresamos nuestro agradecimiento a la Autoridad Nacional de Licencias Ambientales (ANLA), por su colaboración y diligencia durante la recopilación de la información sobre la que se sustenta esta investigación. Este texto publica parte de los resultados obtenidos en la tesis de maestría titulada: "Evaluación de los estudios de mamíferos realizados para el 
Rev. Acad. Colomb. Cienc. Ex. Fis. Nat. 41(158):51-58, enero-marzo de 2017 doi: http://dx.doi.org/10.18257/raccefyn.439

licenciamiento ambiental de proyectos de hidrocarburos en Colombia" (Gómez-Sandoval, 2015). Finalmente, valoramos las observaciones de los pares revisores, que contribuyeron a depurar la versión final de este artículo.

\section{Referencias}

Autoridad Nacional de Licencia Ambientales-ANLA. (2012). Informe de Gestión 2012. Bogotá, D.C. Recuperado de http:/www.anla.gov.co/informes-gestion-autoridad-nacionallicencias-ambientales-anla

Autoridad Nacional de Licencia Ambientales-ANLA. (2014). Informe de Gestión 2013. Bogotá, D.C. Recuperado de http:/www.anla.gov.co/informes-gestion-autoridad-nacionallicencias-ambientales-anla

Autoridad Nacional de Licencia Ambientales-ANLA. (2015). Informe de Gestión 2014. Bogotá, D.C. Recuperado de http://www.anla.gov.co/informes-gestion-autoridad-nacionallicencias-ambientales-anla

Autoridad Nacional de Licencia Ambientales-ANLA. (2015). Informe de Gestión 2015. Bogotá, D.C. Recuperado de http://www.anla.gov.co/informes-gestion-autoridad-nacionallicencias-ambientales-anla

Autoridad Nacional de Licencia Ambientales-ANLA. (2015). Resolución Nº173 del 17 de febrero de 2015 "Por la cual se regula el cobro de copias expedidas por la Entidad”. Bogotá D.C.

Atkinson, S.F., Bhatia, S., Schoolmaster, F.A., Waller. W.T. (2000). Treatment of biodiversity impacts in a sample of US environmental impact statements. Impact Assessment and Project Appraisal. 18: 271-282.

Bataineh, R.H. (2007). The effectiveness of the Environmental Impact Assessment (EIA) follow-up with regard to biodiversity conservation in Azerbaijan. Management of Environmental Quality: An International Journal. 18: 591-596.

Boyles, J.G., Cryan, P.M., MccracKen, G.F., Kunz, T.H. (2011). Economic importance of bats in agriculture. Science. 332: 41-42.

Byron, H.J, Treweek, J.R., Sheate, W.R., Thompson, S. (2000). Road developments in the UK: An analysis of ecological assessment in Environmental Impact Statements produced between 1993 and 1997. Journal of Environmental Planning and Management. 43 (1): 71-97.

Castaño, J. H. \& Corrales, J.D. (2010). Mamíferos de la cuenca del río La Miel (Caldas): diversidad y uso cultural. Boletín Científico Centro de Museos. Museo de Historia Natural. 14: $56-75$.

Ceballos, G., Ehrlich, P.R. (2009). Discoveries of new mammal species and their implications for conservation and ecosystem services. Proceedings from the National Academy of Sciences. 106: 3841-3846.

Fleming, T.H., Geiselman, C., Kress, W.J. (2009). The evolution of bat pollination: a phylogenetic perspective. Annals of Botany. 104: 1017-1043.

Funk, V.A., Hoch, P.C., Prather, A.L., Wagner, W.L. (2005). The importance of vouchers. Taxon. 54: 127-129.

Gómez Sandoval, P.A. (2015). Evaluación de los estudios de mamíferos realizados para el licenciamiento ambiental de proyectos de hidrocarburos en Colombia. (Tesis de Maestría). Universidad Nacional de Colombia. Bogotá.

Gray, I.M., Jones, E.G. (1999). A review of the quality of environmental impact assessments in the Scottish forestry sector. Forestry. 72: 1-10.
Registros de mamíferos en el marco del otorgamiento de licencias ambientales

Hallat, T.W., Retief, F.P., Sandham, L.A. (2015). The quality of biodiversity inputs to EIA in areas with high biodiversity value - Experience from the Cape Floristic Region, South Africa. Journal of Environmental Assessment Policy and Management. 17 (3): 1550025-1-1550025-26.

Isasi-Catalá, E. (2011). Los conceptos de especies indicadoras, paraguas, banderas y claves: su uso y abuso en ecología de la conservación. Interciencia. 36 (1): 31-38

Kageyama, M., Monk, R., Bradley, R., Edson, G., Baker, R. (2006). The changing significance and definition of the biological voucher. En S. Williams \& C. Hawks (ed.). Museum studies: perspectives and innovations. (pp. 259266). Washington: Society for the Preservation of Natural History Collections.

Khera, N., Kumar, A. (2010). Inclusion of biodiversity in environmental impact assessments (EIA): a case study of selected EIA reports in India. Impact Assessment and Project Appraisal. 28 (3): 189-200.

King, N., Rajvanshi, A., Willoughby, S., Roberts, R., Mathur, V.B., Cadman, M., Chavan, V. (2012). Improving access to biodiversity data for, and from, EIAs - a data publishing framework built to global standards. Impact Assessment and Project Appraisal. 30 (3): 148-156.

Kunz, T.H., de Torrez, E.B., Bauer, D., Lobova, T., Fleming, T.H. (2011). Ecosystem services provided by bats. Annals of the New York Academy of Sciences. 1223 (2011): 1-38.

Lee, N., Colley, R. (1990). Reviewing the quality of environmental statements. Occasional Paper 24. Department of Planning and Landscape. University of Manchester. Manchester.

Liévano Latorre, L.F., López Arévalo, H.F. (2015). Comunidad de mamíferos no voladores en un área periurbana andina, Cundinamarca, Colombia. Acta Biológica Colombiana. 20: 193-202.

Mandelik, Y., Dayan, T., Feitelson, E. (2005). Planning for biodiversity: the role of ecological impact assessment. Conservation Biology. 19: 1254-61.

Martin, N.A. (1990). Voucher specimens: a way to protect the value of your research. Biology and Fertility of Soils. 9: 93-94.

Medrano Nájera, R., Ramírez Pinero, M., Guevara Sada, S. (2014). Una mirada a la dispersión de semillas en las excretas de mamíferos. Cuadernos de Biodiversidad. 46 (2014): 19-28.

Mejía Correa, S., Díaz-Martínez, J.A. (2009). Primeros registros e inventario de mamíferos grandes y medianos en el Parque Nacional Munchique, Colombia. Mesoamericana. 13: 7-22.

Ministerio del Ambiente - MINAM. (2015). Guía de inventario de la fauna silvestre. Ministerio del Ambiente. Dirección General de Evaluación, Valoración y Financiamiento del Patrimonio Natural. Ministerio del Ambiente. Lima, Perú. 83 p.m

Ministerio de Ambiente y Desarrollo Sostenible-MADS. (2015). Decreto 1076 de 2015. Por medio del cual se expide el Decreto Único Reglamentario del Sector Ambiente y Desarrollo Sostenible. 653 p.

Morales-Martínez, D.M., Suárez-Castro, A.F. (2014). New records for Glyphonycteris Thomas, 1896 (Chiroptera: Phyllostomidae) from Colombia. Check List. 10: 639-644.

Patton, J.L., Berlin, B., Berlin, E.A. (1982). Aboriginal perspectives of a mammal community in Amazonian Perú: knowledge and utilization patterns among the Aguaruna Jivaro. En: Mares, M. A. \& Genoways, H. H. (ed.). 
Mammalian Biology in South America. (pp. 111-128). Pymatuning Symposia in Ecology 6. Special Publication Series. Pymatuning Laboratory of Ecology. University of Pittsburgh. Pittsburg, Pennsylvania.

Pitman, N., Vriesendorp, C., Alvira, D., Markel, J.A., Johnston, M., Ruelas Inzunza, E., Lancha Pizango, A., Sarmiento Valenzuela, G., Álvarez-Loayza, P., Homan, J., Wachter, T., del Campo, Á., Stotz, D.F., Heilpern, S. (ed.). (2014). Peru: Cordillera Escalera - Loreto. Rapid Biological and Social Inventories Report 26. The Field Museum. Chicago.

Polanco-Ochoa, R., Jaimes, V., Piragua, W. (2000). Los mamíferos del Parque Nacional Natural La Paya, Amazonía colombiana. Revista de la Academia Colombiana de Ciencias Exactas, Físicas y Naturales. 23 (suplemento especial): 671-682.

Proulx, G., Drescher, R.K. (1993). Distribution of the Longtailed Weasel, Mustela frenata longicauda, in Alberta as Determined by Questionnaires and Interviews. Canadian Field Naturalist. 107: 186-191.

Ramírez-Chaves, H.E., Ortega-Rincón, M., Pérez, W.A., Marín, D. (2011). Historia de las especies de mamíferos exóticos en Colombia. Boletín Científico Centro de Museos. Universidad de Caldas. 15 (2): 139-156.

Ramírez-Chaves, H., Suárez-Castro, A.F. (2014). Adiciones y cambios a la lista de mamíferos de Colombia: 500 especies registradas para el territorio nacional. Notas Mastozoológicas. Sociedad Colombiana de Mastozoología. 1 (2): 31-34.

Ramírez-Chaves, H., Suárez-Castro, A.F., González-Maya, J.F. (2016). Cambios recientes a la lista de los mamíferos de Colombia. Mammalogy Notes | Notas Mastozoológicas. 3 (1): $1-9$.

Regan, E.C., Santini, L., Ingwall-King, L., Hoffmann, M., Rondinini, C., Symes, A., Taylor, J., Butchart, S.H.M. (2015). Global Trends in the Status of Bird and Mammal Pollinators. Conservation Letters. 8 (6): 397-403.

Sánchez, F., Sánchez-Palomino, P., Cadena, A. (2004). Inventario de mamíferos en un bosque de los Andes centrales de Colombia. Caldasia. 26: 291-309.

Schilthuizen, M., Vairappan, C.S., Slade, E.M., Mann, D.J., Miller, J.A. (2015). Specimens as primary data: museums and open science. Trends in Ecology and Evolution. 30 (5): 237-238.
Solari, S., Muñoz-Saba, Y., Rodríguez-Mahecha, J.V., Defler, T., Ramírez-Chaves, H., Trujillo, F. (2013). Riqueza, Endemismo y Conservación de los Mamíferos de Colombia. Mastozoología Neotropical. 20 (2): 301-365.

Suarez-Castro, A., Ramírez-Chavez, H., Rodríguez-Posada, M., García, J. (2012). New records of Peropteryx leucoptera and first record of Peropteryx pallidoptera (ChiropteraEmballonuridae) from Colombia. Mastozoología Neotropical. 19: 165-171.

Turney, S., Cameron, E.R., Cloutier, C.A., Buddle, C.M. (2015). Non-repeatable science: assessing the frequency of voucher specimen deposition reveals that most arthropod research cannot be verified. PeerJ. 3: e1168:

Voss, R.S., Emmons, L.H. (1996). Mammalian diversity in neotropical lowland rainforests: a preliminary assessment. Bulletin of the American Museum of Natural History. New York. 230: 1-115.

Voss, R.S., Lunde, D.P., Simmons, N.B. (2001). The Mammals of Paracou, French Guiana: a Neotropical lowland rainforest fauna. Part 2. Nonvolant species. Bulletin of the American Museum of Natural History. 263: 1-236.

Vriesendorp, C., Álvarez, J.A., Barbagelata, N., Alverson, W.S., Moskovits, D.K. (ed.). (2007). Perú: Nanay, Mazán, Arabela. Rapid Biological Inventories Report 18. The Field Museum. Chicago.

Warnken, J., Buckley, R. (1998). Scientific quality of tourism environmental impact assessment. Journal of Applied Ecology. 35: 1-8.

Wheeler, T.A. (2003). The role of voucher specimens in validating faunistic and ecological research. Biological Survey of Canada (Terrestrial Arthropods). Document Series. 9: 1-21.

Zapata-Ríos, G., Araguillin, E., Jorgueson, J.P. (2006). Caracterización de la comunidad de mamíferos no voladores en las estribaciones orientales de la Cordillera del Kutukú, Amazonía Ecuatoriana. Mastozoología Neotropical. 13 (2): 227-238. 